\title{
Designing the Handheld Maritime Communicator
}

Jesper Kjeldskov

Dept. of Computer Science

Aalborg University

Fredrik Bajers Vej 7E

DK-9220 Aalborg East

Denmark

\section{J an Stage}

Dept. of Management Science

and Information Systems

University of Auckland

Private Bag 92019

Auckland, New Zealand

jans@cs.auc.dk jesper@cs.auc.dk

\begin{abstract}
We present the process of designing the first prototype of the Handheld Maritime Communicator: a mobile computer system supporting communication and coordination of safety-critical work activities on large container vessels. Designing the user experience of the Handheld Maritime Communicator was a particular challenge because it targets a highly specialized context of use and because poor design could potentially become a safety hazard. Meeting this challenge, ethnographic field studies on board container vessels were conducted, detailed analyses were carried out, and iterative design was performed. The design produced replaces a large amount of present spoken communication with predefined textual messages on a handheld device. This facilitates persistency, partial automation, and possible integration with other computer-based data. Evaluating the prototype in a high-fidelity ship simulator, prospective users validated the overall design, but also identified a number of usability problems that need to be addressed.
\end{abstract}

\section{Keywords}

Field Studies, Object-Oriented Analysis, I terative Design, Usability Evaluation, Handheld Computing, Text-Based Communication, Collaborative Work, Safety-Critical Use Domains.

for personal or classroom use is granted without fee provided that copies are not made or distributed for profit or commercial

advantage and that copies bear this notice and the full citation on the first page. To copy otherwise, or republish, to post on servers or to redistribute to lists, requires prior specific permission and/or a fee. Copyright 2003, ACM

@ 2003 ACM 1-58113-728-1 03/0006 5.00

\section{I ndustry/ category}

Safety-critical use domains, mobile devices, maritime 


\section{Project statement}

The operation of container vessels in sizes equivalent to $31 / 2$ soccer fields requires workers to be highly mobile and physically distributed. At the same time, work activities on container vessels are often related to the use of computer systems that are centrally located. Recent research has shown that in similar situations where users are concerned with computerized information systems and process control of critical importance remote from their current location, increased utility value can been gained from mobile computers. Examples count the use of remotely controlled service robots for elderly or disabled people [5], distributed process contro in wastewater treatment plants

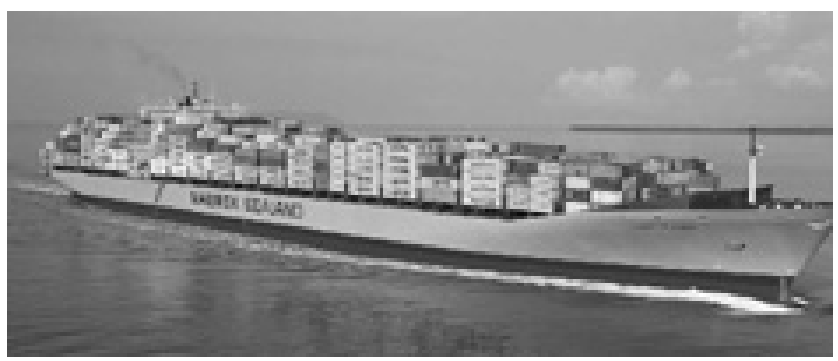

Figure 1: Sally Maersk-one of the world's largest container vessels in operation.
[9], and the use of mobile multimedia communication channels for early diagnosing in emergency ambulance services [14]. In like manner, a strong motivation exists for exploring the use of mobile computers for supporting distributed work activities in the maritime domain.
Designing mobile computer systems for the maritime domain is, however, not trivial. Work activities on large container vessels are typically safety-critical and involve high risks in the case of errors. Especially when maneuvering in narrow or trafficked waters, erroneous actions may cause serious material damage, or possible injuries or even mortalities among personnel.

Therefore, the user experience of mobile systems supporting such work activities has to be carefully designed and evaluated.
In this paper, we describe how a combination of ethnographic field studies, video analysis, objectoriented analysis, and interface design iterations based on simple paper and computer-based mockups informed the design and implementation of the Handheld Maritime Communicator: a prototype application for supporting coordination of distributed collaborative work tasks on board large container vessels.

\section{Project participants}

Four Danish partners participated in the process of designing the Handheld Maritime Communicator:

\$ Aalborg University

\$ Center for Human-Machine I nteraction

\$ Maersk-Sealand

\$ Svendborg International Maritime Academy

A team of researchers from the Center for HumanMachine Interaction (www.chmi.dk) conducted the initial field studies on board large container vessels in collaboration with Maersk-Sealand [1] [2] [10].

Researchers from Aalborg University then conducted additional field studies, carried out video and objectoriented analysis, and designed and implemented the first functional prototype application, which is presented in this paper. The evaluation was done in collaboration between researchers from Aalborg University and Svendborg International Maritime Academy.

\section{Project dates and duration}

To date, the project consisted of five phases. I nitial field studies were conducted in 2000. Data analysis and 


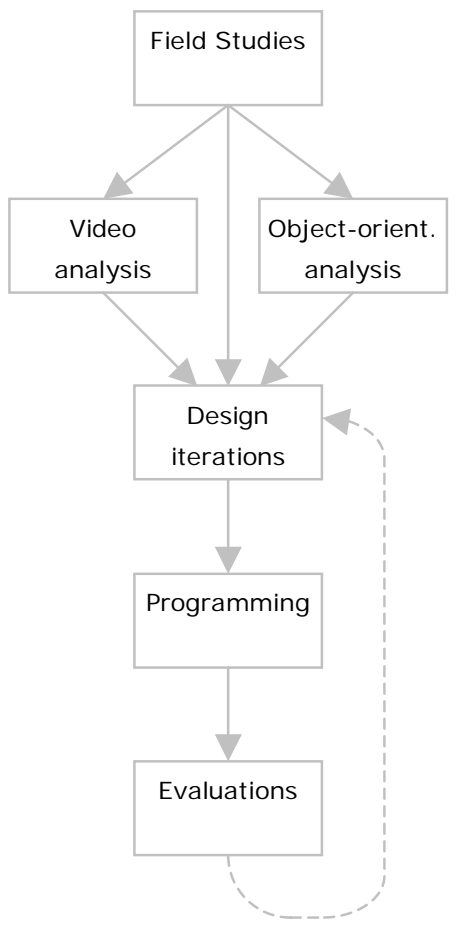

Figure 2: Designing the use experience of the Handheld Maritime Communicator. additional field studies were carried out between August 2001 and May 2002, followed by design and implementation of the Handheld Maritime

Communicator prototype in June and July 2002.

Evaluations were planned and conducted in September and October 2002 and are currently informing the next iteration of design.

\section{Process}

Designing the user experience of the Handheld Maritime Communicator involved the combination of six major activities in a prototyping process:

$\$$ Field studies

$\$ \quad$ Video analysis

$\$$ Object-oriented analysis

$\$$ Design iterations

\$ Implementation of design sketches

$\$$ Usability evaluation of prototype

The specific content and outcome of these activities are described in further details in the following subsections.

As illustrated in Figure 2, the field studies led to video and object-oriented analysis, altogether contributing to a number of design iterations. The design iterations aimed at producing a set of detailed system requirements and a coherent interface concept, which could then be implemented in a functional prototype. At present, the evaluation of the prototype is feeding back into further design iterations.

Field studies

The idea for the Handheld Maritime Communicator originally emerged from a field study on work activities in the maritime domain involving computerized process control and information systems. The field studies were conducted over a period of several months in 2000 and 2001 and involved researchers taking part of a number of voyages on board large Maersk-Sealand container vessels and other ships [1] [10]. During these voyages: data consisting of high-quality video and audio recordings and written notes regarding work activities, communication, and technology use during operation of the vessels; was gathered through observations and interviews in situ.

Apart from informing new interface design for existing maritime instruments [2], a number of work activities were identified in which the use of mobile computer terminals could be desirable. These included diagnostic and maintenance work in the engine room, surveying the condition of reefers during voyages, locating personnel in case of accidents, and supporting various distributed collaborative work activities.

Of particular interest, the field studies provided detailed information on the operation of letting go the mooring lines before departing from harbor and identified some general limitations in present means for communication and coordination in relation to this operation, which could potentially be overcome by the use of mobile computer technology. Supporting this particular operation was, therefore, found to be a suitable starting point for the design of the Handheld Maritime Communicator.

In the following sections, the operation of letting go the lines, as experienced through the field studies, is briefly described. In the subsequent design process, this description served as an overall context for the use of 
the Handheld Maritime Communicator and outlined a number of challenges, which had to be addressed in the design of the user experience.

The operation of letting go the lines

When a container vessel is ready for departure, the first step in leaving the harbor is releasing the mooring holding it in a fixed position [Fig. 3] and heaving the mooring lines onboard the vessel. However, as physical space in harbors is restricted and means for precisely maneuvering large vessels are limited, all lines cannot simply be simultaneously released.

When a line is let go, it will remain in the water for a period of time during which no means of propulsion can be used, due to the risk of lines getting sucked in and wrapped around the propeller or thrusters. Instead, the vessel can be pulled ahead or astern by means of the remaining lines. Following these premises, lines are released sequentially in accordance to the specific need for maneuvering in a given situation.

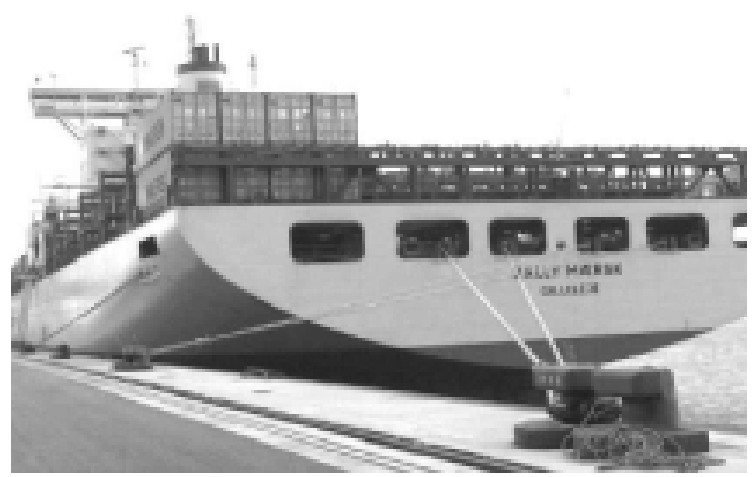

Figure 3: The aft mooring of Sally Maersk.
Due to the huge size of container vessels, the work tasks involved when letting go the lines are distributed among a number of co-workers located at strategic positions [Fig. 4]. On the bridge, chief officers control the rudder, propeller, and thrusters. Fore and aft, the first and second officers control the winches for heaving in the lines. Ashore, two teams of assistants lift the lines off the bollards. To insure the safety of the operation, individual work tasks are carefully coordinated and carried out under strict command of the captain in charge.

At present, communication between co-workers in the maritime domain is primarily spoken. While people on the bridge can see and hear each other directly, personnel on deck are, however, out of direct visual and audio contact and have to communicate with the captain via walkie-talkies.

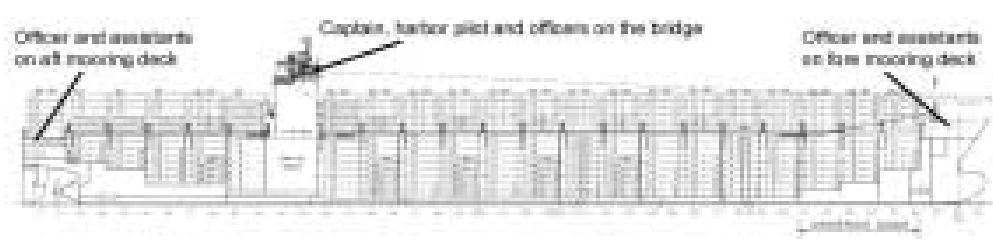

Figure 4: Distribution of co-workers on Sally Maersk during the operation of letting go the lines. 


\section{Key Limitations in Spoken} Communications

Sound quality is often poor Communication is not persistent and...

- Cannot be automated

- Is time consuming

- Suffers from language barriers

- $\quad$ Suffers from bottlenecks (multiple parallel tracks)

- Lacks information integration

For detailed descriptions of these limitations, see [6].
In order to carry out the operation of departure in a safe manner, the captain needs an overview and total control over the propulsion, direction, and mooring of the ship. While information about the rudder, propeller, and thrusters are available on dedicated instruments on the bridge, no information about mooring is facilitated. At present, this only exists as a mental model in the head of the captain. As this mental model is highly sensitive to errors or misunderstandings in the ongoing communication between bridge and deck, and since disparity between the captain's mental model and the real world may cause wrong decisions to be made, considerable resources are spent on establishing and maintaining common ground [4] among the distributed co-workers. Supporting this, well-established rules and procedures exist for oral communication such as, for example, confirming status reports and commands by repeating them back to their sender. To a large extent, these procedures work very well. However, from the observations and interviews, a number of key limitations in the use of spoken communication for coordination of collaborative work activities became apparent (see sidebar).

Overcoming or reducing (at least some of) these limitations served as an overall motivation for the design of the Handheld Maritime Communicator. Inspired by chat applications, newsgroups, and Short Messaging Service (SMS), it was our thesis that shifting to text-based communication on mobile devices would be a feasible approach, because asynchronous textbased messaging is a flexible and persistent communication channel requiring low cognitive overhead [3] [11]. However, we did not know specifically how text-based messaging could be utilized in the design of the Handheld Maritime Communicator's user experience prior to the data analysis.

Video analysis

We had a rich and valuable picture of the operation of letting go the lines, however, designing the user experience of a system for supporting this operation obviously required identification of additional details; for example, how the operation is typically carried out and how it is communicatively coordinated at present. For this purpose, video recordings from the bridge of Sally Maersk during multiple cases of maneuvering inside harbors were transcribed, coded, and analyzed. The video analysis consisted of three steps. First, we carefully examined and discussed the video recordings in relation to the description of the use context outlined previously. This was done to validate our understanding of the overall operation and getting familiar with the events and actions occurring on the video. Secondly, raw transcriptions of communication during the operation of letting go the lines were coded and condensed in order to leave out irrelevant utterances. This was done to capture the essence of communication needed to coordinate and carry out the operation-as exemplified by the following transcription extract of three conversational tracks taking place in parallel.

\section{Transcription extract 1}

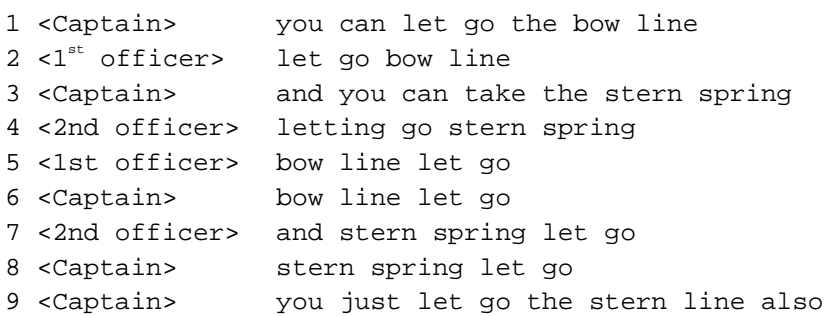


$10<2$ nd officer $>$ let go line aft

$11<1$ st officer> and we have the bow line home

$12<$ Captain>

$13<2$ nd officer $>$ and all let go aft

14 <Captain> all let go aft

Finally, the condensed transcriptions were cut up into smaller sequences and sorted; for example, by their source (who is talking) their subject of reference (who is being talked to), or their object of reference (what is being talked about), as shown below.

\section{Transcription extract 2}

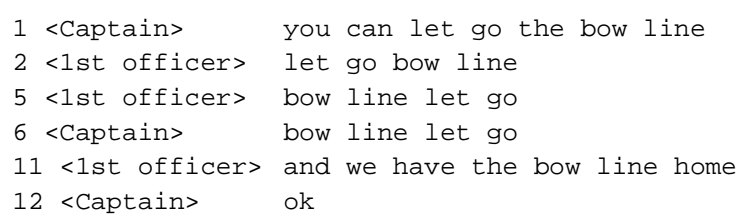

This was done in order to facilitate the possible identification of patterns in current practice, which could potentially be used to support the user experience of a future mobile communication system.

Command sequences and structures in communication The primary outcome of the video analysis was the identification of patterns in the sequence of commands issued by the captain and common structures in the subsequent communication between bridge and deck. Furthermore, a complete set of utterances was produced: commands, confirmations, and status reports necessary for coordinating the whole operation.

Analyzing the typical sequences in the transcriptions of commands issued by the captain revealed that some commands would always precede others. Also, the possible next commands at any point of the operation could always be deduced. We realized that if these sequences were modeled in a computer system, it would be possible to partially automate the overall progression of the operation by, for example, suggesting suitable next steps to the captain. Likewise, organizing the individual utterances of the transcriptions in relation to their objects rather than their temporal sequence (as shown here) revealed that the communication related to each step of the operation followed a common structure, with very little variation. Not surprisingly, this structure was found to correspond to the basics of the "conversation for action" model proposed by speech-act theory [15, pp 65]. We reasoned that if this structure was modeled in a computer system, the current stage of each step of the operation could be formalized and, for example, represented graphically or integrated with other computer-based data. Also, the potential next stages of any step of the operation could be identified and likely utterances by any of the communicating actors at any point of the operation be deduced and possibly prioritized. As subsequent interviews told us that orders were never rejected or withdrawn, only a sub-set of the conversation for action model was implemented.

Object-oriented analysis

At this point of the process, the field study had

identified an overall division of duties of the operation of letting go the lines among a number of distributed co-workers. The video analysis had identified overall sequences and structures in communication. But, our analysis had not yet provided enough information for actually designing a user interface for supporting this operation, nor did we have detailed enough information for modeling and implementing the ideas of 
automation, as previously discussed, that would support the user experience. Therefore, an objectoriented analysis of the problem and application domains of the Handheld Maritime Communicator was conducted using the software engineering method described in [8].

The object-oriented analysis aimed at supporting the design and implementation of the system by identifying objects (classes) and relations in the problem domain and providing detailed descriptions of the states of these classes during the operation. The two major activities of the object-oriented analysis were the modeling of a class diagram for the problem domain and a state chart diagram for the Let Go class.

\section{Class diagram}

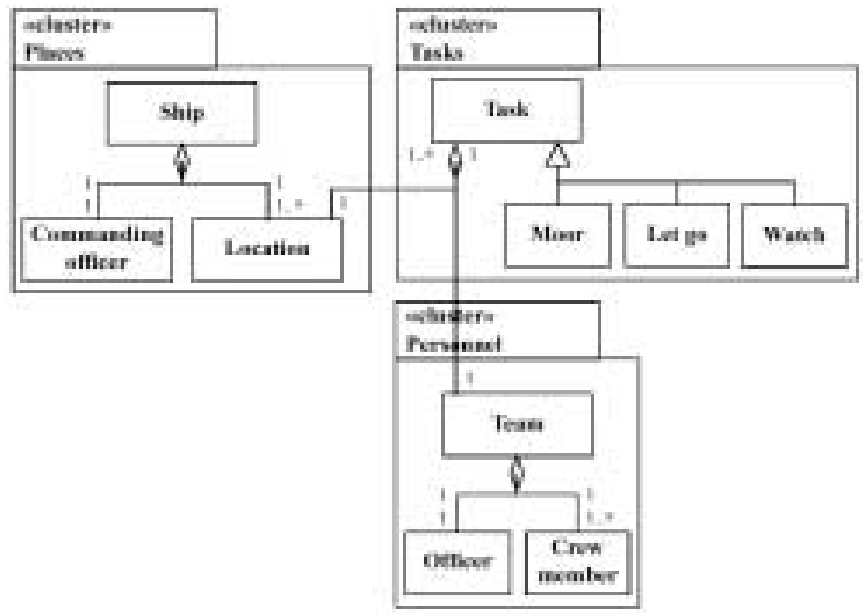

Figure 5: Class diagram for the problem domain
Modeling the class diagram [Fig. 5] was a challenge, primarily because we extended the problem domain to include work activities related to maneuvering inside the harbor other than just the operation of letting go the lines. This was done to support extending the scope of the Handheld Maritime Communicator later, without having to change the underlying model. Doing this, however, required us to reexamine the video recordings and include classes and relations, which would not be of direct importance to letting go the lines.

From the class diagram, we identified a number of issues that directly informed the design of the user experience. First, the class diagram illustrated that letting go the lines is one of three similar operations (or tasks) related to maneuvering inside the harbor. On the basis of this, we found that the user experience of the Handheld Maritime Communicator might benefit from facilities for tailoring an interface supporting each of these specific operations, on the basis of a general design for supporting communication and coordination. Thus, general interface elements should be associated to the overall Task class, while more specialized interface elements, such as specific graphical representations, should be associated with their appropriate specialization.

Second, we found that since the Commanding Officer class and the Officer class aggregate from

fundamentally different classes (Ship and Team, respectively), different interfaces for these actors might also be appropriate. Thus the interface for the commanding officer should possibly include information about the ship as a whole, while the interface for the officers on deck should instead possibly include information about the Team.

Finally the class diagram helped us realize the role of the Location class as a mediator between the Ship and Task classes. Knowing the location of a mobile device would enable us to deduce the role of its user in an ongoing task and adapt the interface accordingly. 
State chart diagram

As the Let Go class would contain the structure of the operation and communication, a lot of effort was put into modeling its state chart [Fig. 6]. Even though valuable input for this already existed from the video analysis, making a precise model was a challenge. First, a number of variations in the sequence of the operation had to be modeled. Second, we had to model that depending on the situation: some commands implicitly contained others. Finally, we had to include the possibility of commands being executed in parallel.

The state chart diagram for the Let Go class provided a detailed view of the operation of letting go the lines.

First of all, it provided both the details and abstraction needed to actually implement the partial automation of sequence and communication discussed previously. Second, it showed that each command goes through three overall temporal stages of being: (1) imminent (future tense), (2) executing (present tense), and (3) ended (past tense). While in some situations ended commands may be important, whereas in other situations only executing commands are vital, dividing the interface into three corresponding, overall areas reflecting this categorization might support the user experience by enabling simple differentiation of priority.

Design iterations

Following the analysis, a design team consisting of the authors of this paper engaged in a series of iterations aimed at producing specific requirements for the interface design and user experience of the Handheld Maritime Communicator.

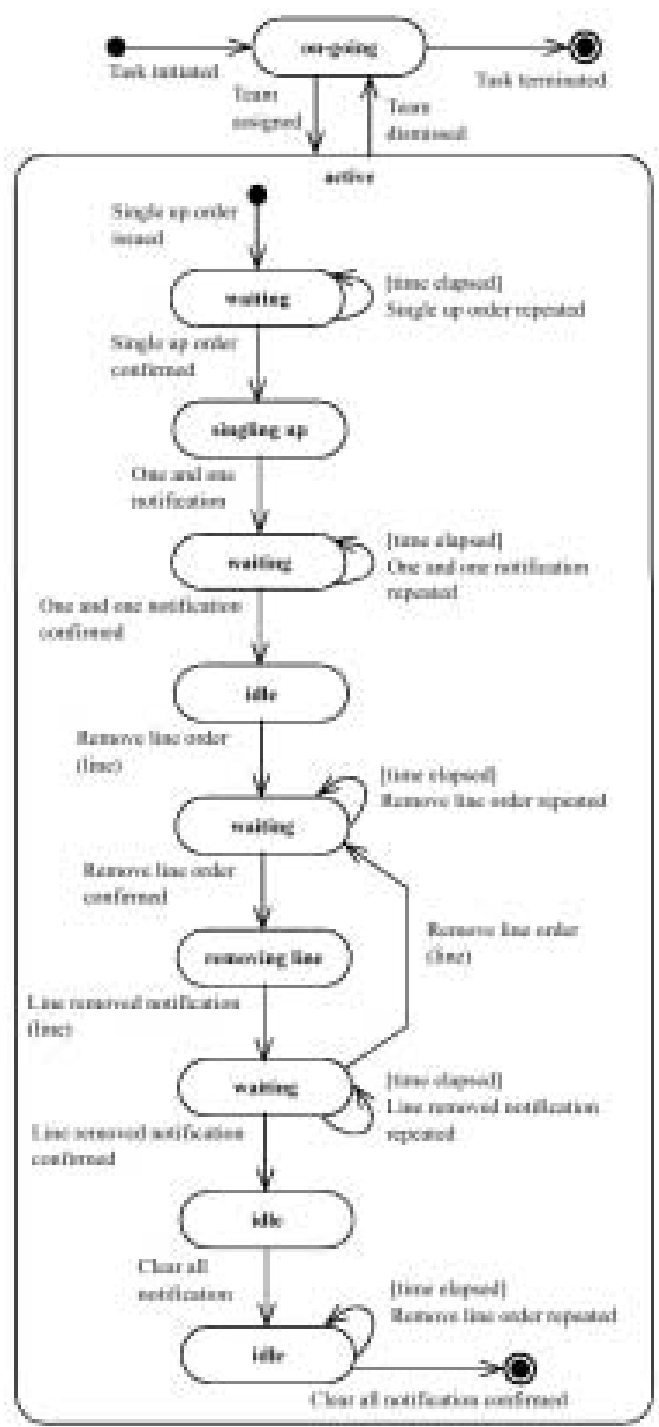

Figure 6: State chart diagram for the Let Go class. 
As described in the following sessions, three iterations of design were carried out, each producing design documents and interface sketches on different levels of detail. Apart from being informed by the outcome of the field study, video analysis, and object-oriented analysis, the designs produced during the iterations were inspired by literature on supporting text-based communication such as [11], [12], and [13], and preceding design of text-based communication systems such as chat-applications, newsgroups, and Short Messaging Service (SMS).

Paper-based mockups

First, a series of paper-based mockups of possible interface designs were produced on the basis of the analysis previously described and inspired by the use of multi-threaded communication and predefined messages in other systems. These paper mockups facilitated fundamental discussions of the basic interface design of the Handheld Maritime Communicator and led to an overall concept for the user experience providing (1) a graphical representation of the ship and its mooring lines, (2) a transcription of the communication sorted by objects of reference, and (3) a facilitation for communication through predefined textual commands selected from lists.

Visual Basic sketches

On the basis of the paper mockups, detailed design sketches were produced in Microsoft eMbedded Visual Basic. This forced the design team to work within the limitations of the target platform in terms, for example, of supported screen size and graphical interface elements. Consequently, a number of shortcomings arose related to the specific division of screen real estate and the desired level of details of graphical representations. While most of the subsequent refinements of the design were done directly in Visual Basic, larger design issues, such as how to support the textual representation of multiple parallel threads of commands in what turned out to be a very limited graphical space, temporarily forced the design team back to pen and paper. Eventually, a final Visual Basic design sketch was produced.

Shockwave prototype

While the Visual Basic based design sketches did not have any functionality, screen dumps modified in Adobe Photoshop were used in the third design iteration to produce a Shockwave based prototype in Macromedia Director showing a possible sequence of communication. Adding life to the Visual Basic design sketches in this "quick-and-dirty" fashion facilitated further discussions and resulted in minor modifications of the design before doing any actual programming. For example, it was decided that the user should not be prompted for confirmations through pop-up screens (as these would temporarily cover vital information). Instead, confirmations should be included in a dropdown menu containing all other commands.

Implementation of design sketches

After a final design had been agreed upon, this design was implemented in a functional prototype, using Microsoft eMbedded Visual Basic. The implementation process consisted of five phases. First, the underlying data structure of the system was implemented in accordance to the class diagram and state charts produced in the object-oriented analysis. Following this, the user interface was implemented in accordance to the final design sketches. When the prototype was 


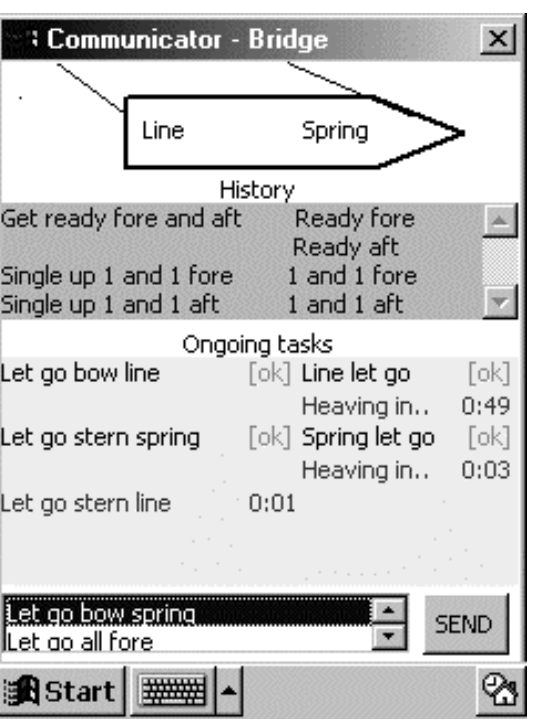

Figure 8: The interface on the bridge. working properly, network interfaces and protocols were implemented for enabling the distribution of the system on multiple devices. For the first prototype, we used a wireless TCP/IP network. Given the use domain, however, this will probably not be a viable solution for the final application due to radio interference from the hull and the risk of losing network packages. Finally, a configuration screen was implemented, and extensive code testing and minor bug fixes were performed.

\section{Solution details}

The following sections describe the design of the Handheld Maritime Communicator.

\section{Hardware}

The Handheld Maritime Communicator prototype was designed to run on three Compaq IPAQ 3630

\section{Architecture}

The application running on the captain's device works as a server containing a formalized representation of the operation to be supported and the typical pattern of communication. The devices on deck log on to this server and report their physical location, after which an appropriate interface is displayed on them. During the operation, function calls and unique command identifiers are exchanged over the network, eliminating the problem of commands being missed in the air due to poor sound quality. All network communication is broadcast, but processed and represented differently on each device, in accordance with its physical location. Also, as a unique feature, the desired language can be defined individually on each device, thereby reducing potential language barriers between co-workers by automatically translating commands. Language is specified in a simple text file.

handheld computers connected through a wireless network. One device was intended for the captain on the bridge while the other two were intended for first and second officers on the fore and aft deck, respectively. Apart from a touch screen, the IPAQ facilitated interaction by means of a five-way key located below the display and suitable for onehanded interaction. Due to the potentially harsh conditions of the use domain, in which pen-based interaction might be problematic, we decided that all interaction should be facilitated by the use of this key. Thus, moving the five-way key left or right selects different elements on the screen, whereas moving the key up or down browses the items of lists. Clicking on the center of the key selects a highlighted item.
The user experience

Reducing bottlenecks and supporting persistence in communication, the Handheld Maritime Communicator prototype gives the distributed actors on the container vessel access to a mobile, text-based communication channel and provides a graphical representation of the ship and its mooring lines.

The overall interface depicted in Figure 8 is divided into four sections. The three last correspond to the temporal stages of commands identified in the analysis:

$\$$ Pictogram of the ship and mooring lines

$\S$ List of completed commands

$\$$ List of ongoing commands

$\$$ List of possible commands 
At the bottom of the screen, standard commands are displayed on a list (figure 9). The order of the list corresponds to the standard sequence of the overal operation and commands only appear when appropriate. By default, the most likely next step of the operation is highlighted. Commands can be browsed and executed (send) with the five-way key.

(a)

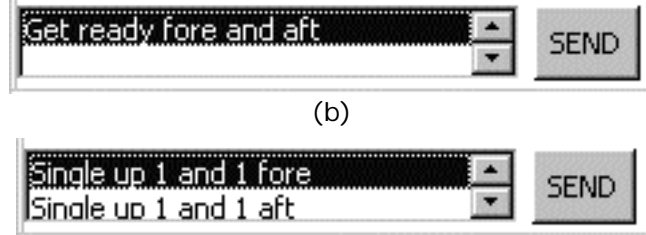

Figure 9: Executed commands being removed from the command list while new commands appear.

When a command is executed, it is removed from the list, and the most likely next step is highlighted. A unique feature of this design is that interaction required by the captain during standard procedures is limited to a minimum. An immediate constraint of this design, however, is the limited flexibility in the sequence of the operation facilitated as well as limited means for communicating something out of the ordinary.

Perhaps the most important element of the interface is the list of ongoing tasks. Here, inspired by newsgroups and multi-threaded chat applications, ongoing threads of communication are represented textually, grouped in accordance to the object to which they refer. Displaying parallel communication threads textually this way reduces the bottlenecks observed when multiple people speak simultaneously. When a new command is executed, it appears on this list. Next to it, a counter displays the time passed while waiting for confirmation
[Fig. 10a]. When a command is confirmed, the timer is substituted by the text "ok," in brackets, followed by a description of the current activity (e.g., "Singling

up..."). A counter next to this displays the time passed since confirmation [Fig. 10b]. When a task is reported completed, a short statement (e.g., "1 and 1 fore") substitutes for the description of activity, and the captain is prompted for confirmation [Fig.10c]. Completion of a task is confirmed, indicated by the text "ok" [Fig. 10d]. As a unique feature of this design, the list of ongoing tasks anticipates and makes space for additional steps in each thread and displays not only raw communication but also the present status of each command being executed. Again, an immediate constraint of this design is the lack of flexibility

(a)

Ongoing tasks

Single up 1 and 1 fore 0:02

Single up 1 and 1 aft $0: 01$

(b)

Ongoing tasks

Single up 1 and 1 fore [ok] Singling up.. $\quad 0: 01$

Single up 1 and 1 aft [ok] Singling up.. $\quad 0: 03$

(c)

Ongoing tasks

Single up 1 and 1 fore [ok] 1 and 1 fore [ ]

Single up 1 and 1 aft [ok] Singling up.. $\quad 3: 10$

(d)

Ongoing tasks

Single up 1 and 1 fore [ok] 1 and 1 fore [ok]

Single up 1 and 1 aft [ok] 1 and 1 aft [ ]

Figure 10: Commands being executed (a), confirmed (b), completed (c), and confirmed (d). 
When the captain confirms the completion of a task, it is removed from the list of ongoing tasks and added to the history list [Fig. 11]. As a unique feature, this list automatically filters itself to contain only the initiating commands and the subsequent outcome, thereby reducing the complexity of the user experience. When the history list is full, it automatically scrolls the oldest commands and statements out of immediate sight.

(a)

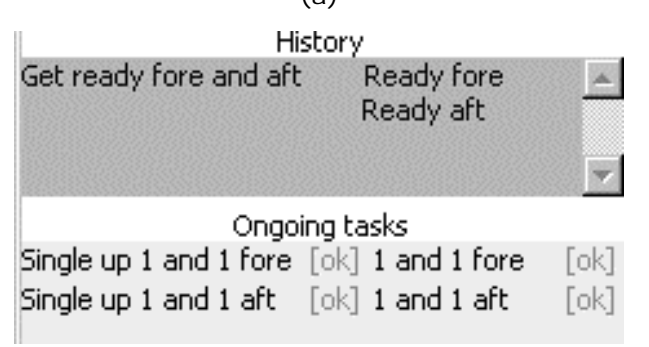

(b)

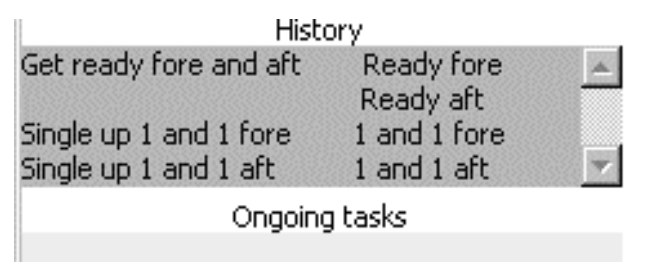

Figure 11: Completed commands being transferred to the history list

Another unique feature of the design is the formalized graphical representation of the mooring lines currently attached to the quay based on the status of the ongoing communication. While only containing redundant information that can also be deduced from the textual descriptions on the display, the graphical representation facilitates an overview of the current situation, which is not supported in current practice.

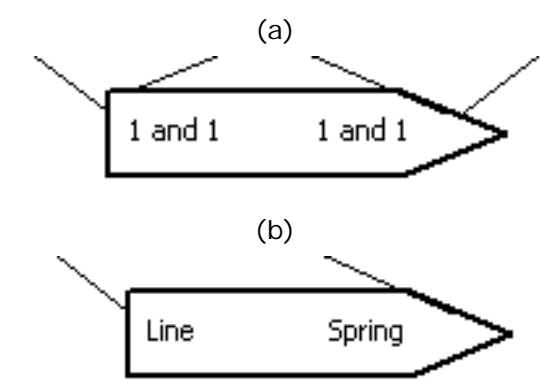

Figure 12: Visual representations of mooring lines

The interfaces on deck are very similar. In the list of ongoing tasks, however, users are requested to confirm commands by the captain such as "Let go bow spring." Correspondingly, the list of predefined commands only contains those appropriate at the specific location at given states of the operation, e.g., "Confirm: Let go bow spring" for confirmation of the latter command or "Spring let go" for reporting the completion of this task.

\section{Results}

The first functional prototype aimed at facilitating additional refinements of the design through userbased evaluations. Due to the nature of the use domain, however, evaluating the user experience of the prototype was a challenge for a number of reasons.

First of all, evaluating the system in the real world was not possible at the present level of implementation due to safety issues. Secondly, personnel from the observed container vessels were a scarce resource for evaluation purposes. Therefore, for the purpose of studying the experience of the prototype in the hands of prospective users in a realistic, yet safe and controllable 


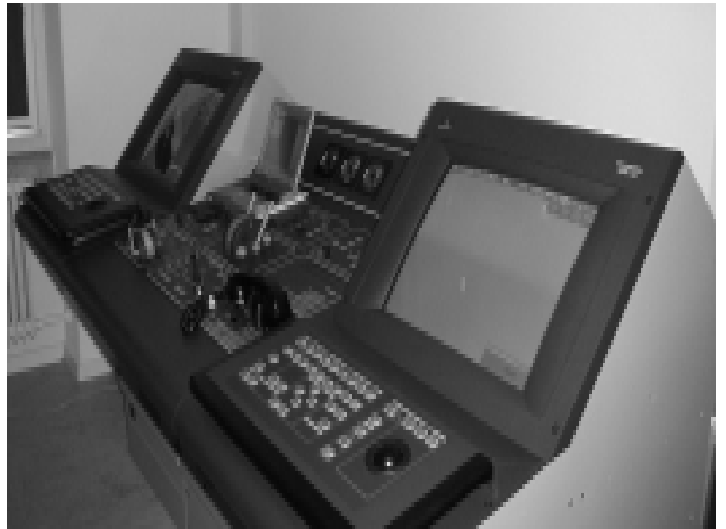

environment, collaboration was set up with Svendborg I nternational Maritime Academy to use their state-of-the-art ship simulator and staff of experienced maritime officers [7]. The design of this study is described in the following sections, followed by a discussion of its outcome.

\section{User study design}

Setting up the ship-simulator user study involved developing a challenging yet realistic use scenario and programming this into the simulator system. Also,

Figure 13: Controls on the simulated bridge.

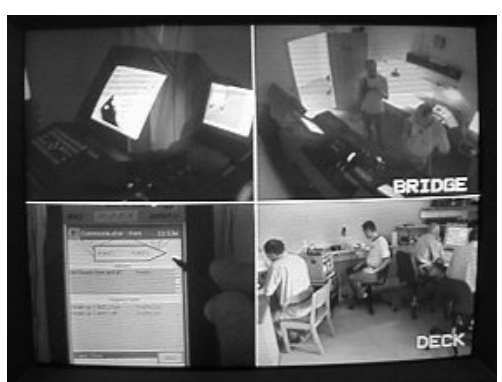

Figure 14: Video recording of use in the ship simulator. prospective users had to be profiled and assigned to the evaluation sessions. This was done in collaboration with the simulator division of Svendborg International Maritime Academy. Finally, a temporary usability laboratory setup had to be established facilitating the physical separation of test subjects, and dynamically feeding information from the ongoing communication between bridge and deck back into the simulation. Also, high-quality video and audio recordings of the sessions had to be enabled for later analysis. Whereas the preliminary preparations took about a week, the evaluation itself was conducted in a single day.

The ship simulator was set up to imitate the operation of a large vessel in challenging weather and traffic conditions, corresponding to a real-world situation observed during our field studies [10].

Three teams, of two trained maritime officers each, were given the task of letting go the lines and departing from harbor using the Handheld Maritime Communicator for communication between bridge and deck. One user acted as captain on the simulated bridge [Fig. 13], while the other acted as first officer on the fore deck in the neighboring simulator control room. For simplicity, commands targeted at the second officer on the aft deck were fed directly into the simulation, and feedback was given by the simulation operator. Carrying out the operation, the user acting as captain had to consider all aspects of maneuvering the ship. This included controlling the rudder, propellers, and thrusters, as well as communicating with personnel on the ship, harbor traffic control, and so on; and taking into consideration the movements of other vessels. The primary tasks of the first officer on deck were to orally forward commands executed by the captain via the mobile device prototype to the operator of the simulation (impersonating the team of assistants carrying out the actual tasks) and to report back to the captain. The operator would then enter the commands into the simulation and report to the first officer when the requests (such as letting go a line) had been carried out.

During the evaluation, the users were asked to think aloud, explaining their experience of and interaction with the prototype. Two evaluators, located on bridge and deck, observed the users and asked questions for clarification. Following each evaluation session, a group interview of 10-15 minutes was carried out.

Total views of the bridge and deck/simulator control room, as well as close-up views of the mobile devices, were captured by four video cameras and merged into one high-quality video signal providing a synchronized view of the whole setup [Fig. 14]. Audio from the two rooms was recorded on separate tracks for possible separation during playback for analysis. 
Outcome from user study

We analyzed the videotapes to identify design aspects and to create a list of any usability problems. Observing the experience of the prototype in the ship simulator (reported as a positive experience by the users) provided rich data on the usability of the design.

First of all, the user study showed that the prototype could actually replace the majority of spoken

communication between the captain and officers. Also, the users told us that the text-based interface gave them a simple channel of communication in which they could easily overview the ongoing communication and progress of the operation. Generally, the users learned what to do and what feedback to expect within the completion of one or two threads of communication, reporting that the design was very intuitive to use. The differentiation between future and present commands appeared straightforward, as well as the grouping and progress of ongoing threads. For quick overview, the graphical representation of the ship, not provided in current practice, was highly appreciated, and wishes for more details were expressed. On the contrary, the history list was rarely used.

On the negative side, the user study also revealed 22 usability problems on different levels of seriousness experienced by more than one user. First of all, a need for requesting or reporting something out of the ordinary and correcting or withdrawing statements was identified. The decision to implement only a sub-set of the conversation for the action-communication model [15] turned out to limit the usability of the design. Also, a number of standard commands, such as dismissing a team or requesting a status report, were missing. All users, at some point of the operation, decided to call the other user on the radio. In one situation, for example, a wrong command was executed due to interaction problems and could not be withdrawn. Therefore, the captain had to correct the error over the radio. Feedback on the counter order also could not be carried out textually in the system. In another situation, the ship had been brought into a situation in which the remaining line was about to break. The officer on deck was aware of this problem, but had no means of reporting it textually to the captain. When requested to let go the particular line, the system did not let him report that the request could not be met (and why), and the officer thus had to use the radio. Facilities for supporting this communication could easily be included in the system by extending the underlying model of communication structure.

Secondly, we observed that while all users executed commands or reports straightforwardly, it was unclear how to confirm the reception of these. Most expected to find this functionality in the list of ongoing tasks and no one immediately noticed the appearance of a

highlighted confirm option in the list of commands. One user completely misinterpreted this option and did not want to use it. Furthermore, we observed that pending confirmations often went unnoticed by the captains. One captain never confirmed reports from deck until four or five had piled up. Another did not confirm anything. On deck, this lack of feedback caused doubt about whether or not reports had been successfully received. Finally, some officers on deck expressed that while textual communication supported overview,

having to pull out the device and look at it for reading a command was not ideal. Having commands read by a synthetic voice was suggested as a supplement to the interface. 


\section{Acknowledgements}

The authors thank Peter Bøgh Andersen, Center for HumanMachine Interaction, Svendborg International Maritime Academy, and all test subjects. The usability evaluation sessions were planned conducted, and analyzed in collaboration with Mikael B. Skov.
At present, the outcome of the user study is informing redesign of the prototype. While the overall interface design was validated and it was proved possible and useful to replace a large proportion of spoken communication by predefined text-based messages, the ongoing iteration of design focuses on supporting more flexibility by fully implementing the conversation for action model [15] and differentiating more clearly between commands and confirmations. Furthermore, the use of multi-modal interaction is being investigated as a supplement to the textual interface. We intend to evaluate the next iteration of design onboard real container vessels in operation. Generally, the process described illustrates the value of a rich understanding of the use context, as well as powerful tools for modeling, when designing user experiences for mobile device interfaces that support a specialized work task.

\section{References}

11] Andersen, P. B. Communication and work on maritime bridges. CHMI Research report CHMI-1-2000 http://www.cs.auc.dk/ pba/ElasticSystems (2000).

[2] Andersen, P. B. and May, M. Tearing up Interfaces. In Liu K. et al (eds.) Information, organization and technology. Studies in organizational semiotics. Boston, Klüwer (2001).

[3] Churchill E. F. and Bly S. It's all in the words: Supporting work activities with lightweight tools. In Proceedings of ACM Siggroup'99 (1999), 40-49.

[4] Clark, H.H. and Schaefer, E. F. Contributing to discourse. Cognitive Science, 1989, 13, 259-294 (1989).

[5] Hüttenrauch, H. and Norman, M. PocketCERO - mobile interfaces for service robots. In Proceedings of MobileHCl'01, IHM-HCl, Lille, France (2001).

[6] Kjeldskov J. and Stage J. Designing the User Interface of a Handheld Device for Communication in a High-Risk
Environment. In Adjunct Proceedings of the ERCIM 2002 Conference on Universal Access, Paris, France (2002).

[7] Kjeldskov J. and Skov M. B. Evaluating the Usability of a Mobile Collaborative System: Exploring Two Different Laboratory Approaches. In Proceedings of Symposium on Mobile Technology for Collaborative 2003, Orlando, Florida (2003).

[8] Mathiassen, L., Munk-Madsen, A., Nielsen, P. A., and Stage, J. Object-Oriented Analysis and Design. Aalborg, Marko (2000).

[9] Nielsen, C. and Søndergaard, A. Designing for mobility evaluated-an integration approach supporting multiple technologies. In Proceedings of NordiCHI 2000, 23-25 October 2000, Royal Institute of Technology, Stockholm, Sweden (2000).

[10] Nielsen, $M$. Letting go the lines: Departure from the Felixstowe harbor. CHMI Research report CHMI-4-2000 http: //www.cs.auc. dk/ pba/ElasticSystems (2000).

[11] Popolov, D. Callaghan, M. and Luker, P. Conversation Space: Visualising Multi-threaded Conversation. In Proceedings of AVI 2000, Palermo, I taly, ACM (2000), 246-249.

[12] Smith M., Cadiz J. J. and Burkhalter B. Conversation Trees and Threaded Chats. In Proceeding of CSCW'00, Philadelphia, USA, ACM Press (2000), 97-105.

[13] Smith M. A. and Fiore A. T. Visualization Components for Persistent Conversations. In Proceedings of $\mathrm{CHI} \mathrm{01,}$ Seattle, USA, ACM Press (2001), 136-143.

[14] van den Anker, F. W. G. and Lichtveld, R. A. Early Evaluation of New Technologies: The Case of Mobile Multimedia Communications For Emergency Medicine. In Vincent C. and de Mal B (eds.) Safety in Medicine. Qxford: Elsewier Science (2000).

[15] Winograd T. and Flores F. Understanding Computers and Cognition. Addison-Wesley (1986). 